\title{
Efficient Assignment of Multiple E-MBMS Sessions towards LTE
}

\author{
Antonios Alexiou $^{1}$, Christos Bouras ${ }^{1,2}$, and Vasileios Kokkinos ${ }^{1,2}$ \\ ${ }^{1}$ Computer Engineering and Informatics Dept., Univ. of Patras, Greece \\ ${ }^{2}$ Research Academic Computer Technology Institute, 26500 Rio, Patras, Greece \\ alexiua@ceid.upatras.gr, bouras@cti.gr, kokkinos@cti.gr
}

\begin{abstract}
One of the major prerequisites for Long Term Evolution (LTE) networks is the mass provision of multimedia services to mobile users. To this end, Evolved - Multimedia Broadcast/Multicast Service (E-MBMS) is envisaged to play an instrumental role during LTE standardization process and ensure LTE's proliferation in mobile market. E-MBMS targets at the economic delivery, in terms of power and spectral efficiency, of multimedia data from a single source entity to multiple destinations. This paper proposes a novel mechanism for efficient radio bearer selection during E-MBMS transmissions in LTE networks. The proposed mechanism is based on the concept of transport channels combination in any cell of the network. Most significantly, the mechanism manages to efficiently deliver multiple E-MBMS sessions. The performance of the proposed mechanism is evaluated and compared with several radio bearer selection mechanisms in order to highlight the enhancements that it provides.
\end{abstract}

\section{Introduction}

Nowadays, mobile industry rapidly evolves towards a multimedia-oriented model for providing rich services, such as Mobile TV and Mobile Streaming. LTE networks address this emerging trend, by shaping the future mobile landscape. However, the plethora of mobile multimedia services that are expected to face high penetration, poses the need for deploying a resource economic scheme.

In order to confront such high requirements for multimedia content, LTE networks rely on the E-MBMS framework. E-MBMS constitutes the evolutionary successor of MBMS, which was introduced in the Release 6 of Universal Mobile Telecommunication System (UMTS) [1], [2]. The main requirement in E-MBMS services is to make an efficient overall usage of radio and network resources. This necessity mainly translates into improved power control strategies, since the base stations' transmission power is the most limiting factor of downlink capacity. Under this prism, a critical aspect of E-MBMS performance is the selection of the most efficient radio bearer for the transmission of multimedia traffic.

In the frame of power control and transport channel selection during multimedia data delivery, several approaches have been proposed. The 3rd Generation Partnership Project (3GPP) TS 25.346 [3] and TR 25.922 [4], as well as work [5] are representative approaches. However, all of these works focus on MBMS, without considering E-MBMS and LTE requirements. 
In this paper, we propose a novel radio bearer selection mechanism for E-MBMS. The proposed scheme adopts downlink transmission power as the optimum criterion for radio bearer deployment and selects the transport channel combination that minimizes the transmission power of the base station. Therefore, Point-to-Point (PTP) and Point-to-Multipoint (PTM) transmission modes may be used separately or be combined and deployed in parallel. However, the most remarkable advantage of the proposed mechanism, that actually differentiates it from the above approaches, is that it may simultaneously serve multiple multimedia sessions. Our approach is compared with the 3GPP approaches in terms of power consumption and complexity so as to highlight its enhancements and the necessity for its incorporation in E-MBMS specifications.

The paper is structured as follows: In Section 2, we present the motivation behind our study and the related work in the specific field. Section 3 presents the proposed power control mechanism, while Section 4 is dedicated to the presentation of the results. Finally, the planned next steps as well as the concluding remarks are briefly described in Section 5.

\section{Motivation and Related Work}

The transmission of MBMS packets over the air interfaces may be performed on common (Forward Access Channel or FACH), dedicated (Dedicated Channel or DCH) or shared channels (High Speed-Downlink Shared Channel or HS-DSCH). Each channel has different power consumption characteristics [6], [7], [8].

The selection of the most efficient bearer is still an open issue in today's MBMS infrastructure, mainly due to its catalytic role in Radio Resource Management (RRM). The following paragraphs present the main radio bearer selection approaches existing in the bibliography.

\subsection{MBMS Counting Mechanism (TS 25.346)}

The 3GPP MBMS Counting Mechanism (TS 25.346) constitutes the prevailing approach of switching between PTP (multiple DCHs) and PTM (FACH) radio bearers, mainly due to its simplicity of implementation and function [3]. According to this mechanism, a single transport channel can be deployed in a cell at any given time. The decision on the threshold between PTP and PTM bearers is operator dependent, although it is proposed that it should be based on the number of served MBMS users. In other words, a switch from PTP to PTM resources should occur, when the number of users in a cell exceeds a predefined threshold. However, this mechanism provides a non realistic approach because mobility and current location of the mobile users are not taken into account. Moreover, this mechanism does not support FACH dynamic power setting. In other words, when FACH is employed, it has to cover the whole cell area that generally leads to unnecessary power wasting. Finally, TS 25.346 does not support the HSDPA technology, which could enrich MBMS with broadband characteristics [6].

\subsection{MBMS PTP/PTM Switching Algorithm (TR 25.922)}

3GPP TR 25.922 or MBMS PTP/PTM switching algorithm [4], assumes that a single transport channel can be deployed in a cell at any given time. Contrary to TS 25.346, 
it follows a power based approach when selecting the appropriate radio bearer, aiming at minimizing the base station's power requirements during MBMS transmissions. In TR 25.922, instead of using solely DCHs, HS-DSCH can also be transmitted. However, the restricted usage of either DCH or HS-DSCH in PTP mode may result to significant power losses. In both cases, the PTP (DCH or HS-DSCH, since the switching between HS-DSCH and DCH is not supported in this mechanism) and the PTM power levels are compared and the case with the lowest power requirements is selected. Even though TR 25.922 overcomes several inefficiencies of the TS 25.346, it does not support FACH dynamic setting.

\subsection{Mechanism Proposed in 3GPP TSG RAN1 R1-02-1240}

All the above mechanisms allow a single PTP or PTM transport channel deployment at any given time. On the other hand, the mechanism proposed in 3GPP TSG RAN1 R1-02-1240 [5], considers the mixed usage of DCHs and FACH, which can significantly decrease the base station's transmission power, depending on the number and the location of the users. According to this approach, the FACH channel only covers a dynamically selected inner area of a cell and provides the MBMS service to the users that are found in this part. The rest of the users are served using $\mathrm{DCH}$ to cover the remaining outer cell area.

However, none of the above MBMS power control mechanisms takes into account the ability of the base stations to support many simultaneous MBMS sessions. MBMS transmissions have increased power requirements and consume a large portion of the available power recourses of the base stations. Consequently, the number of parallel MBMS sessions that a base station could support is limited. Therefore, the selection of the appropriate radio bearer for a MBMS service should be done with respect to other existing MBMS sessions in the corresponding cell. The number of parallel MBMS sessions that a base station could support depends on many parameters. We could classify these parameters in three categories: user related parameters, MBMS session related parameters and provider related parameters. User related parameters are parameters such as UEs' (User Equipment) distances from the base stations and UEs' Quality of Service (QoS) parameters. The number of active MBMS sessions per cell, the number of UEs per MBMS session per cell and the bit rates of the MBMS services are some of the MBMS session related parameters. Finally, the portion of the available power recourses of base stations that could be used for MBMS transmissions is a provider related parameter. All these parameters should be considered in the RRM of MBMS so as to have efficient power control.

\section{Proposed Mechanism for PTP and PTM Bearers Combination}

This section presents the architecture and the functionality of the MBMS session assignment mechanism. The proposed mechanism incorporates all the basic functionalities of the standardized 3GPP approaches and furthermore, it integrates several enhancements (power based transport channel selection, combined usage of transport channels, parallel MBMS sessions and user mobility support). 


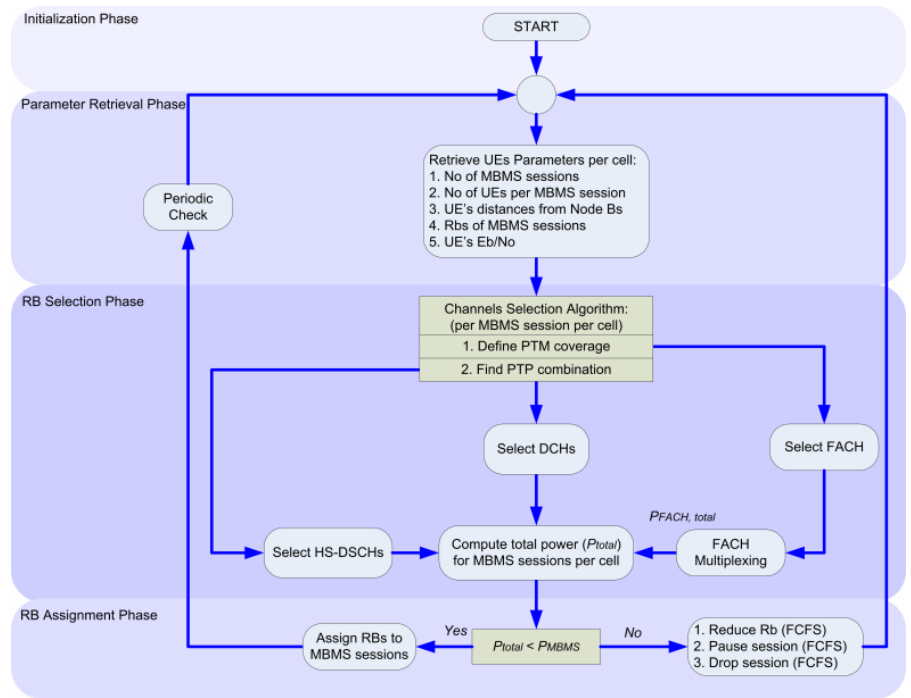

Fig. 1. Block diagram of the mechanism

The block diagram of the mechanism is illustrated in Fig. 1. According to Fig. 1, the mechanism consists of four distinct operation phases. These are: the initialization phase, the parameter retrieval phase, the radio bearer $(\mathrm{RB})$ selection phase and the $\mathrm{RB}$ assignment phase.

The initialization phase (Fig. 1) launches the mechanism when one user expresses his interest in receiving a MBMS service (i.e. the mechanism begins when the first user requests the first MBMS service).

The parameter retrieval phase is responsible for retrieving the parameters of the existing MBMS users and services in each cell. In this phase, the mechanism requires the two of the three types of parameters, mentioned in the previous section: the user related parameters and the MBMS session related parameters. Regarding the latter type of parameters, the mechanism requires information about the number of active MBMS sessions per cell, the number of UEs per MBMS session per cell and the bit rates of the MBMS sessions. This information is retrieved from the Broadcast Multicast - Service Center (BM - SC). On the other hand, user related parameters are retrieved from the UEs through uplink channels.

The RB selection phase is dedicated to the selection of the transport channels for the MBMS sessions in any cell of the network. The most critical operations of the phase are executed by the Channels Selection Algorithm block (Fig. 1). The algorithm executed in this block selects the combination of PTP and PTM bearers that minimizes the downlink base station's transmission power in any cell of the network that multicast users are residing. In particular, the algorithm is executed in two steps. In the first step (Define PTM coverage) the algorithm estimates the optimum coverage of FACH for the users' distribution of any MBMS session in the cell. This coverage area is called inner part of the cell as illustrated in Fig. 2. In the second step (Find PTP combination), the mechanism decides which PTP bearer(s) will cover the rest 


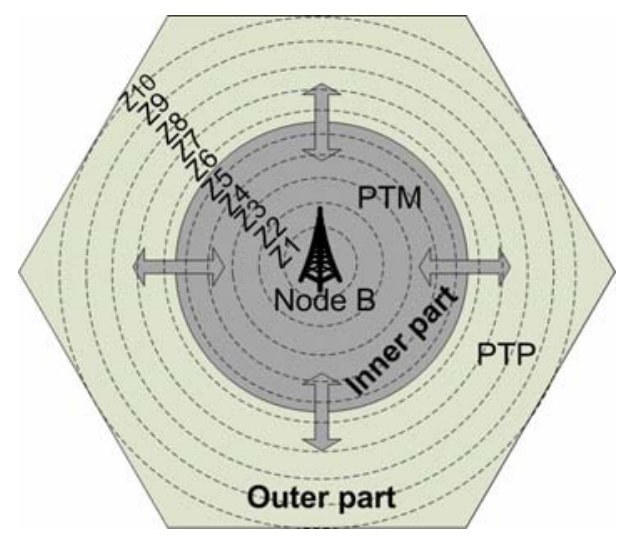

Fig. 2. Cell areas and zones

part of the cell (outer part - Fig. 2). It has to be mentioned that the above cell characterization is done for every MBMS session of the corresponding cell.

In order to estimate the optimum coverage of FACH (for any MBMS session in the cell) in Define PTM coverage step (Fig. 1), the algorithm initially divides the cell in ten zones (Z1 to Z10) according to the FACH dynamic power setting technique. Each zone $\mathrm{Zi}$ refers to a circle with radius equal to $10 \mathrm{i} \%$ of cell radius. Afterwards, the algorithm scans all the zones and calculates the total base station's transmission power for the following 21 transport Channel Configurations (CC):

- CC1: No FACH used. All users are covered by DCHs.

- CC2: No FACH used. All users are covered by HS-DSCHs.

- CC3: FACH for UEs up to Z1. All the rest UEs covered by DCHs.

- CC4: FACH for UEs up to Z1. All the rest UEs covered by HS-DSCHs.

$-\ldots . . . . . .$.

- CC19: FACH for UEs up to Z9. All the rest UEs covered by DCHs.

- CC20: FACH for UEs up to Z9. All the rest UEs covered by HS-DSCHs.

- CC21: FACH for all UEs (up to Z10). DCHs and HS-DSCHs are not used.

The $\mathrm{CC}$ that consumes less power indicates the coverage of the FACH and determines the inner part of the cell. The same procedure is executed for any MBMS session in the cell. The output of the Define PTM coverage step is the coverage of the FACH for any MBMS session in the examined cell.

Once the appropriate FACH coverage for any MBMS session in the cell is defined, the algorithm enters the Find PTP combination step (see Fig. 1), which determines the appropriate PTP radio bearer(s) that will cover the MBMS users residing in the outer part of the cell for any MBMS session. The procedure is similar to the procedure described in the Define PTM coverage step. The algorithm scans all the zones in the outer part of the cell and calculates the total base station's transmission power in order to cover all the outer part MBMS users only with PTP bearers. The first zone of the outer part is Z(inner part+1), therefore the algorithm will have to scan the following PTP transport Channel Configurations (PTP_CC): 
- PTP_CC1: DCHs for outer part UEs up to Z(inner part+1). All the rest outer part UEs (up to Z10) covered by HS-DSCHs.

- PTP_CC2: DCHs for outer part UEs up to Z(inner part+2). All the rest outer part UEs (up to Z10) covered by HS-DSCHs.

- PTP_CC(10-inner part): All MBMS users in the outer part cell are covered by DCHs. HS-DSCHs are not used.

- PTP_CC(10-inner part+1): HS-DSCHs for outer part UEs up to Z(inner part+1). All the rest outer part UEs (up to Z10) covered by DCHs.

- PTP_CC(10-inner part+2): HS-DSCHs for outer part UEs up to Z(inner part+2). All the rest outer part UEs (up to Z10) covered by DCHs.

- PTP_CC $(2 *(10$-inner part)): All MBMS users in the outer part cell for the specific session are covered by HS-DSCHs. DCHs are not used.

After these calculations, the different PTP_CCs are compared and the PTP_CC with the lowest power requirements determines the PTP transport channel configuration for the outer part MBMS UEs of the specific MBMS session in the cell. The procedure is recursively executed for any MBMS session in the cell.

Generally, the output of the Channels Selection Algorithm block is the combination of PTM and PTP transport channels that consumes the lowest power resources between all possible combinations in the corresponding cell for any MBMS session running in it.

In the case of FACH there is another block in the mechanism's block diagram named FACH Multiplexing. When the number of MBMS sessions requiring FACH in cell is greater than one, these FACHs should be multiplexed onto a Secondary Common Control Physical Channel (S-CCPCH) [9], [10]. After the multiplexing procedure, the capacity of the S-CCPCH is calculated and based on this, the total power required for the common channels $\left(P_{F A C H, \text { total }}\right)$ in the corresponding base station is estimated. In this paper we consider a one to one mapping between MBMS sessions and FACHs.

The last action performed in the RB selection phase is the computation of the total base station's power $\left(P_{\text {total }}\right)$ required to support all the MBMS sessions in each cell. However, we have to mention that the selected radio bearers are not yet assigned to the MBMS sessions. This action is performed in the following phase.

During the RB assignment phase, the $P_{\text {total }}$ is compared with the available power assigned by the network provider to MBMS sessions in each base station $\left(P_{M B M S}\right)$. Obviously, the $P_{M B M S}$ constitutes the third type of parameters mentioned in the previous section, known as provider related parameter. If $P_{\text {total }}$ is smaller than $P_{M B M S}$, the selected from the RB selection phase transport channels are assigned to MBMS sessions and the MBMS data transfer phase begins. In case when $P_{\text {total }}$ is bigger than $P_{M B M S}$, a session reconfiguration procedure should occur due to the fact that there are no available radio resources to the base station so as to serve all the MBMS sessions in the examined cell. In this paper, we propose three possible reconfiguration events that could be used in such a case. The first is the reduction of the transmission rate of a MBMS session, the second is the pause of a MBMS session for a short time period and the last is the service cancellation. 
The simplest policy in order to perform the three above reconfiguration events, is a First Come First Served (FCFS) policy. Following the FCFS policy and considering the available power, the mechanism performs the optimum event to the most recent MBMS sessions.

The above description refers to a dynamic model, in the sense that the UEs are assumed to be moving throughout the topology and the number of MBMS sessions varies. The parameter retrieval phase is triggered at regular time intervals so as to take into account the user related parameters, the MBMS session related parameters and the operator related parameters. This periodic computation inserts a further complexity as this information is carried in through uplink channels. This entails that a certain bandwidth fraction must be allocated for the transmission of this information in uplink channels, thus resulting to a system's capacity reduction. A further complexity is inserted due to the fact that the mechanism is executed many times for each cell in the topology.

\section{Performance Evaluation}

In this section, analytical simulation results for the evaluation of the mechanism are presented. In particular, through two different scenarios we examine the following key aspects of the mechanism (efficiency, comparison with current 3GPP approaches, handling of multiple parallel MBMS sessions).

The main assumptions of our simulations are presented in Table 1 and refer to a macrocell environment [8], [10]. In addition, Block Error Rate (BLER) target is set to $1 \%$ and no Space Time Transmit Diversity (STTD) is assumed.

Table 1. Simulation assumptions

\begin{tabular}{ll}
\hline Parameter & Value \\
\hline Cellular layout & 18 hexagonal grid cells \\
Sectorization & 3 sectors/cell \\
Site-to-site distance & $1 \mathrm{Km}$ \\
Maximum BS Tx power & $20 \mathrm{~W}(43 \mathrm{dBm})$ \\
Other BS Tx power & $5 \mathrm{~W}(37 \mathrm{dBm})$ \\
CPICH power & $2 \mathrm{~W}$ \\
Common channel power & $1 \mathrm{~W}(30 \mathrm{dBm})$ \\
Propagation model & Okumura Hata \\
Multipath channel & Vehicular A $(3 \mathrm{~km} / \mathrm{h})$ \\
Orthogonality factor & 0.5 \\
$E_{b} / N_{0}$ target & $5 \mathrm{~dB}$ \\
\hline
\end{tabular}

\subsection{Comparison with 3GPP Approaches}

The first scenario lasts for $200 \mathrm{sec}$ and can be divided into four time periods, depending on the number of MBMS users. According to this scenario, a $64 \mathrm{Kbps}$ service should be delivered to a group of users, whose initial position at each time period is presented in Table 2. For example, for the period 0 to $50 \mathrm{sec}, 25$ UEs receive the service at distance $50 \%$ and 7 UEs at distance $80 \%$ of the cell radius. 
Table 2. UE Number, Coverage per time period

\begin{tabular}{llll}
\hline $\begin{array}{l}\text { Time } \\
(\mathrm{sec})\end{array}$ & $\begin{array}{l}\text { UEs } \\
\text { No }\end{array}$ & $\begin{array}{l}\text { Coverage } \\
(\%)\end{array}$ & $\begin{array}{l}\text { Best } \\
\text { Performance }\end{array}$ \\
\hline $0-50$ & 25 & 50 & Our Mechanism \\
& 7 & 80 & \\
$51-100$ & 25 & 50 & R1-02-1240, Our Mechanism \\
& 2 & 80 & \\
$101-150$ & 17 & 50 & TR 25.922 (HS-DSCH), Our Mechanism \\
$151-200$ & 4 & 50 & All except TR 25.922 (HS-DSCH) \\
\hline
\end{tabular}

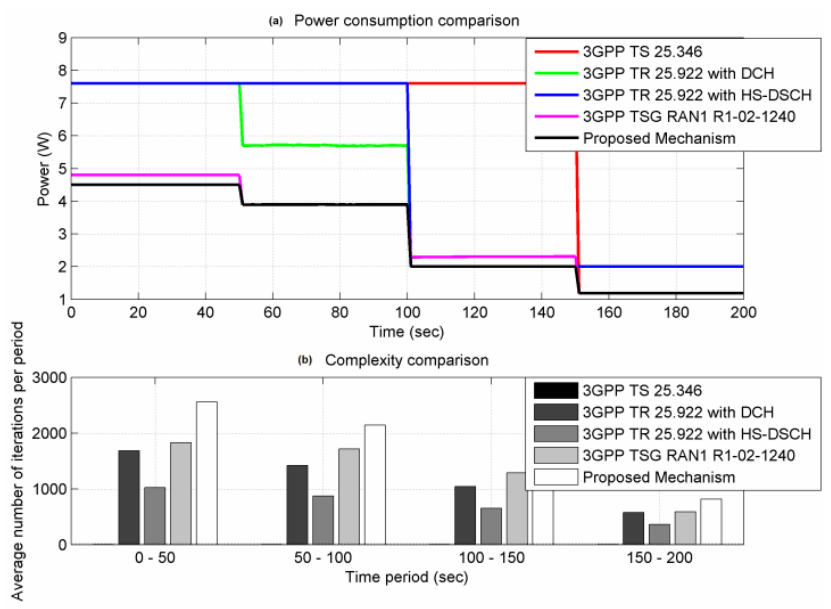

Fig. 3. (a) Power consumption and (b) complexity comparison

Fig. 3a depicts the power levels of the examined approaches. For example, for the period 0-50 sec, the total number of users in the cell is 32. By assuming that the threshold for switching between DCH and FACH (HS-DSCH is not supported) in TS 25.346 is $8 \mathrm{UEs}$ (a value proposed in the majority of research works), TS 25.346 will deploy a FACH with $100 \%$ cell coverage (requiring $7.6 \mathrm{~W}$ ).

The high initial users' population favors the deployment of FACH in order to serve all the UEs in 3GPP TR 25.922. However, as TS 25.346, TR 25.922 does not support FACH dynamic setting. This is the reason why TR 25.922 has the same power requirements with TS $25.346(7.6 \mathrm{~W})$ for the time period 0-50 sec.

The mechanism proposed in RAN1 R1-02-1240 allows the mixed usage of DCHs and FACH and supports FACH dynamic power setting. As shown in Fig. 3a, this mechanism requires $4.8 \mathrm{~W}$ in order to serve all the users, for the first time period. More specifically, this mechanism will deploy only a FACH with $80 \%$ coverage, since the user with the worst path loss resides at the borders of zone $\mathrm{Z8}$.

Finally, Fig. 3a depicts the power requirements of the proposed mechanism. For the time period 0-50 sec, the output of the Channels Selection Algorithm block (Fig. 1) specifies that the users up to $\mathrm{Z5}$ should be served by a FACH. Moreover, the most efficient combination of PTP bearers for the outer part MBMS users is to serve the 
remaining 7 users in zone $\mathrm{Z} 8$ with HS-DSCH. Therefore, $4.5 \mathrm{~W}$ in total are required in order to serve all the MBMS users with this mechanism. Obviously, a significant power budget, ranging from 0.3 to $3.1 \mathrm{~W}$, may be saved for the period $0-50 \mathrm{sec}$ compared with the other approaches.

On the other hand, Fig. $3 \mathrm{~b}$ presents the computational overhead that each mechanisms inserts (number of iterations required to calculate the power and assign the ideal channel), based on the above scenario. In general, TS 25.346 inserts the lowest computational overhead, since it requires only the number of served MBMS users in order to assign the appropriate transport channel. The other approaches have higher computational overhead due to the fact that these mechanisms have to periodically retrieve the parameters of existing MBMS users. Moreover, these approaches have to calculate the power consumption of the supported transport channels; and based on this calculation to assign the ideal radio bearer. The fact that the proposed mechanism supports all the available channels and examines all possible transport channels configurations explains why its computational overhead is higher than the other approaches.

To sum up, the proposed mechanism outperforms the other approaches in terms of power consumption, since significant power budget is saved. It puts together the benefits of all mechanisms by providing a scheme that is based on the concept of transport channels combination. And even if the complexity of the proposed mechanism is higher than the complexity of the other mechanisms, the benefits from the optimal power planning counterbalance the complexity issues raised.

\subsection{Managing Parallel MBMS Sessions}

In order to evaluate the ability of the proposed mechanism to manage multiple parallel MBMS sessions, we setup a simulation scenario where multiple MBMS services are transmitted in parallel to several user groups residing in a cell. In particular, we suppose that four user groups receive four distinct MBMS services with characteristics presented in Table 3. Moreover, Table 3 presents the appropriate transport channel (with respect to power consumption) to serve each group at each time interval. Fig. 4 depicts the power consumption of each MBMS session as well as the total, aggregative power required to support the transmission of all services to the multicast users in the corresponding cell.

Users of the 1st MBMS session are served with a HS-DSCH channel, due to the small population, throughout the whole service time. At simulation time $50 \mathrm{sec}$, MBMS service 2 is initiated (Fig. 4). At this time instant, the mechanism, through the

Table 3. Scenario parameters

\begin{tabular}{llllll}
\hline $\begin{array}{l}\text { MBMS } \\
\text { No. }\end{array}$ & $\begin{array}{l}\text { Duration } \\
(\mathrm{sec})\end{array}$ & $\begin{array}{l}\text { Bit } \\
\text { Rate }\end{array}$ & $\begin{array}{l}\text { UEs } \\
\text { Number }\end{array}$ & $\begin{array}{l}\text { Maximum } \\
\text { Coverage }\end{array}$ & Channel \\
\hline 1 & $0-600$ & 64 & 10 & $80 \%$ & HS-DSCH \\
2 & $50-600$ & 64 & 22 & $60 \%$ & FACH \\
3 & $100-150$ & 64 & $2-13$ & $60 \%$ & DCH \\
& $151-300$ & 64 & $14-19$ & $60 \%$ & HS-DSCH \\
& $301-600$ & 64 & $20-27$ & $60 \%$ & FACH \\
4 & $150-560$ & 64 & 7 & $70 \%$ & DCH \\
& $561-600$ & 32 & 7 & $80 \%$ & DCH \\
\hline
\end{tabular}




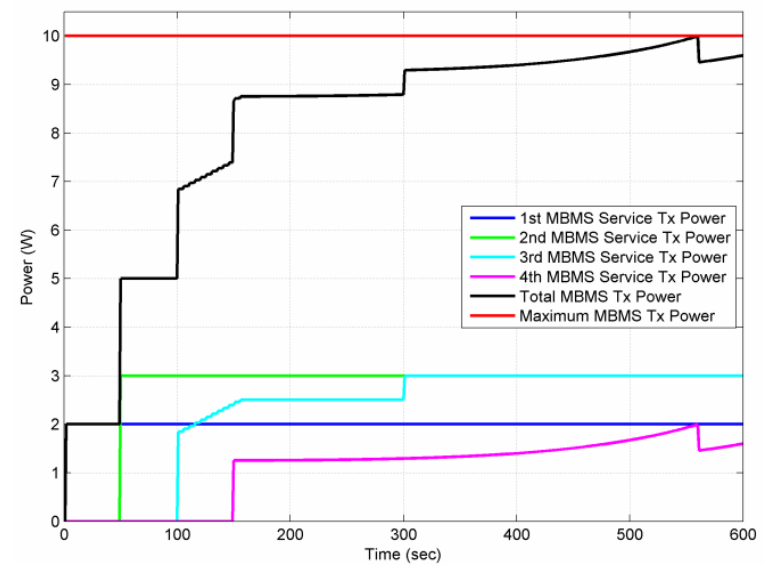

Fig. 4. Power levels of the MBMS sessions

RB selection phase, selects FACH as the most efficient transport channel for the transmission of the second MBMS traffic, since MBMS session 2 is delivered to a large number of users (22 UEs).

MBMS service 3 starts at simulation time $100 \mathrm{sec}$. At this time the $3 \mathrm{rd}$ multicast group consists of only two UEs; thus, the mechanism selects multiple DCHs for this MBMS service. The number of users receiving the service successively increases (join requests), reaching 13 UEs at simulation time $150 \mathrm{sec}, 19$ at $300 \mathrm{sec}$ and 27 at the end of the simulation. The increasing number of users in the group forces the mechanism to perform a channel switching from DCH to HS-DSCH at simulation time $151 \mathrm{sec}$ and another one from HS-DSCH to FACH at simulation time $301 \mathrm{sec}$, securing the efficient resource utilization.

At this point we have to mention that from time $300 \mathrm{sec}$ until the end of the simulation, MBMS services 2 and 3 employ FACHs for the transmission of the MBMS data (see Table 3). During this time interval, the deployment of two parallel FACHs forces the mechanism to perform a FACH multiplexing procedure in the RB selection phase. Consequently, a single $\mathrm{S}-\mathrm{CCPCH}$ with bit rate of $128 \mathrm{Kbps}$ is used to deliver services 2 and 3. Additionally, $P_{\text {total }}$ is lower than $P_{M B M S}$, which translates into efficient provision of the three parallel MBMS sessions.

At simulation time $150 \mathrm{sec}$, the MBMS service 4 is initiated and is targeted to a multicast group consisting of seven members. Multiple DCHs are selected by the mechanism to deliver the MBMS content to the 4th multicast group. Additionally, at the same time instance, $P_{\text {total }}$ still remains smaller than $P_{M B M S}$, which means that the MBMS service 4 is accepted for transmission in the system. From simulation time 150 until the end of the simulation, four parallel MBMS sessions running in the system and our mechanism handles them in an efficient way.

Due to the fact that the users of the 4th multicast group are moving towards the cell edge an increase in $P_{\text {total }}$ occurs and at simulation time $560 \mathrm{sec}$; and $P_{\text {total }}$ exceeds $P_{M B M S}$ value (Fig. 4). Thus, a session reconfiguration procedure is performed, forcing 
the MBMS service 4 to reduce its bit rate from $64 \mathrm{Kbps}$ to $32 \mathrm{Kbps}$ in order to ensure the efficient service of four parallel MBMS sessions without any interruption.

\section{Conclusions and Future Work}

In this paper we presented a novel power control mechanism for efficient radio bearer selection in E-MBMS enabled networks. The proposed mechanism adopts the concept of radio bearer combination (PTP and/or PTM) so as to reduce the power requirements of the base stations and shares efficiently the available power resources of base stations to MBMS sessions running in the network. In order to highlight the enhancements obtained by the proposed mechanism, we provided a comparison of the mechanism with current 3GPP approaches and other works. The main conclusion is that our mechanism outperforms them in terms of power consumption, underlining the necessity for its incorporation in E-MBMS.

The steps that follow this work could be at a first level the evaluation of the mechanism through additional simulation scenarios so as to measure other parameters such as delays in air interfaces during MBMS transmissions. At a second level, we plan to improve the functionality of our mechanism by incorporating the enhancements obtained from the use of multiple-input multiple-output (MIMO) antennas in High Speed Downlink Packet Access (HSDPA).

\section{References}

1. 3GPP TS 22.146 V8.3.0. Technical Specification Group Services and System Aspects; Multimedia Broadcast/Multicast Service; Stage 1 (Release 8) (2007)

2. 3GPP TR 23.846 V6.1.0. Technical Specification Group Services and System Aspects; Multimedia Broadcast/Multicast Service; Architecture and functional description (Release 6) (2002)

3. 3GPP TS 25.346 V8.1.0. Technical Specification Group Radio Access Network; Introduction of the Multimedia Broadcast Multicast Service (MBMS) in the Radio Access Network (RAN); Stage 2, (Release 8) (2008)

4. 3GPP TR 25.922 V7.1.0. Technical Specification Group Radio Access Network; Radio resource management strategies (Release 7) (2007)

5. 3GPP TSG-RAN WG1\#28 R1-02-1240. Power Usage for Mixed FACH and DCH for MBMS, Lucent Technologies (2002)

6. Holma, H., Toskala, A.: HSDPA/HSUPA for UMTS: High Speed Radio Access for Mobile Communications. John Wiley \& Sons, Chichester (2006)

7. Perez-Romero, J., Sallent, O., Agusti, R., Diaz-Guerra, M.: Radio Resource Management Strategies in UMTS. John Wiley \& Sons, Chichester (2005)

8. 3GPP TR 25.803 V6.0.0. Technical Specification Group Radio Access Network; S$\mathrm{CCPCH}$ performance for MBMS (Release 6) (2005)

9. 3GPP TS 25.211 V7.4.0. Physical channels and mapping of transport channels onto physical channels (FDD) (Release 7) (2007)

10. 3GPP TS 25.212 V8.0.0. Multiplexing and channel coding (FDD) (Release 8) (2007)

11. 3GPP TR 101.102 V3.2.0. Universal Mobile Telecommunications System (UMTS); Selection procedures for the choice of radio transmission technologies of the UMTS (UMTS 30.03 version 3.2.0) 